\title{
Disposed to Acts
}

\section{GREg NOBLE}

UNIVERSITY OF WESTERN SYDNEY

\author{
Bernard Lahire \\ The Plural Actor \\ Translated by David Fernbach \\ Polity, Cambridge 2010 \\ ISBN 9780745646855 \\ RRP \$37.95
}

In the preface to this English translation of his 2001 book, L'homme pluriel, Bernard Lahire describes how he'd planned this work as a 'theoretical parenthesis', a 'clarification' following years of empirical research, but it became an extended engagement with sociological problems, particularly those emanating from the work of Pierre Bourdieu. I wish I could write 'clarifications' like this. Lahire's writing has rarely been translated into English, so this is a welcome opportunity to see something of his extensive body of work.

The book is divided somewhat theatrically into four 'acts', each of which has two or more 'scenes'. Act I: Outline of a Theory of the Plural Actor takes its cue very obviously from Bourdieu. Lahire argues against 'singleness' and homogeneity (of 
culture and individual): the tendency for researchers to see individuals as representatives of groups, and thus to display the totality of characteristics of that group. (11-12) At the same time, however, Lahire criticises those approaches which celebrate the fragmentation of human identities. He argues for the necessity of examining the complexities and specificities of the socio-historical conditions of someone's dispositions.

To address this, Lahire takes as his starting point an interrogation of Bourdieu's notion of habitus - the embodied dispositions of perception and actionbut in terms of a recognition of the multiplicity of the social worlds, institutions and situations in which we act, and their varying intensities and regularities. Lahire is no Bourdieu lackey: he is critical of 'servile disciples' as much as those who want to relegate him to the sociological past, and aims for a reinvention of Bourdieu's project. (xiv) Lahire argues, for example, that Bourdieu's notions of habitus and disposition need to work at the level of the embodied individual, not just as an abstraction. He posits 'a sociology on the individual scale', a 'psychological sociology': not an individualist model, but one which avoids reducing humans to ciphers for social categories and which explores each human, action and situation as complexly social. (xii-xiii)

This approach opens up Bourdieu's emphasis on the durability of embodied dispositions to explore the tendency for scholarship to oscillate between an oversocialised and an undersocialised habitus. The coherence or otherwise of someone's habitus, therefore, 'depends on the coherence of the principles of socialisation to which they have been subjected'. (25) The plurality of social contexts produces repertoires of habit. Lahire insists he is offering 'a sociology that is indissociably both dispositional and contextual', taking into account both the embodied past and the different present contexts of action. (xi) To see the significance of this we need to acknowledge that Bourdieu's notion of habitus is at the same time a theory of socialisation, a theory of action, and a theory of practice. (ix)

Crucially, there is no 'unifying principle or generating formula' to account for all practices, or both individuals and groups. Practices can only be understood as 'the intersection of embodied dispositions ... and contextual constraints' which produces social actors who must be 'dispositionally plural' because each situation 
does not simply require that we 'apply' our dispositions, but that we can act appropriately in a plurality of contexts. (xi) Lahire decouples Bourdieu's relation between habitus and field, arguing that social worlds are only sometimes organised as fields or structured spaces of positions, citing the family as the prime example. The theory of fields, he claims, may solve some conceptual problems, but it creates others. (28-9) Lahire is also critical of Bourdieu's privileging of those occasions where there is a 'happy' fit, and argues that maladjustment, discrepancy, contradiction, transplanting and rupture are much more typical of social existence. (46-7)

Using a more diverse array of contexts than field theory, Lahire characterises an individual as the depository of diverse dispositions that are the product of multiple socialising experiences, in various collectives; individuals, then, are not reducible to a single collective, but defined by range of competing cultural influences: 'we live experiences that are varied, different and sometimes contradictory'. We are many things at once: this is what Lahire means by the plurality of the actor. (31) This leads him to emphasise the embodiment of each actor in terms of a multiplicity of schemes of action, repertoires of behaviours specific to each context, which we are capable of moving between.

Central to the way these issues are framed is the problem of the relationship between past and present in action. Humans are neither actors without pasts or actors whose behaviour is simply determined by their past; we need a more nuanced approach to understanding this relationship and the 'wellsprings of action'. It is the 'conditional' nature of dispositions which Lahire draws on to provide this nuance, using research on code-switching and code-mixing to illustrate humans' capacities to possess plural habits. (61) He explores a range of practices-such as practical analogy, involuntary action, habits, sensory triggers of memory-to consider both the ways practices are always the 'meeting point' of past experiences (embodied as schemes of action) and current situations, (66) and the ways they problematise our understanding of the 'transferability' of dispositions across situations. (77) Schemas of action are often partial, and their transfer conditional, not generalisable and determining. (83)

Lahire is no abstract theoretician-his work comes out of sustained empirical research. This research-on literacy, the 'double life' of writers, class 
practices of reading, education-poses particular conceptual problems. Act II: Reflexivities and Logics of Action begins with a discussion of schools, but this is no dry educational researcher talking. It begins with his disarming claim that what happens at schools 'is weird and astonishing' because it entails a 'radical ontological transformation' in our relationship to language; in contrast to the being 'in' language emphasised by phenomenologists, we hold language in front of us, requiring reflection, distance, abstraction and analysis. (101-2) In one of the quirkier chapters which has resonances both with ethnomethodology and cultural studies' analysis of mundane behaviour, Lahire examines everyday practices of writing, including the embodied memory of the 'domestic' writing of shopping lists. He argues that such everyday practices offer symbolic mastery of what had previously been mastered practically: time, space and language. But he also argues more that such writings are 'daily exceptions to the pre-reflexive adjustment of practical sense to a social situation'; a rupture with the practical logic of performance in the 'obviousness of things to be done'. (121-2) He contrasts these with his discussion of crises which demand readjustment, but he also suggests that the distance offered through everyday writing provides a space for reflection and planning which is distinct from the 'spontaneity' of language in face-to-face encounters. (131) Such practices allow people to shape the temporalities of their existence, to constitute a future. (139) Lahire uses these cases to problematise the universality of the habitus, and a tendency to reduce habit, and habitus, to the non-reflexive kind. (145) Teasing out aspects of Bourdieu's work, the distinction between practical mastery and symbolic mastery, or between the 'practical' and 'reflexive' modes of habitus, allows Lahire to think about the ambiguity of practice, the plurality of times and actions, and the multiplicity of the social worlds we inhabit. In making these distinctions, the book reasserts what Bourdieu neglected-the capacity for reflexion in everyday activities. Lahire is critical of Bourdieu's emphasis on the singular nature of both 'logic' and 'practice' and the phenomenological preoccupation with the isolated moment of an action. He is scathing of the 'epistemological error' common in the social sciences of generalising from a particular variety of cases to produce a 'perfect' but singularising theory that cuts out all the complication of human practice, its elongated timeframes beyond the moment of action, the drawing in and out of reflexivity, correction and rehearsal, and the pedagogical presence of others. (148ff) 
This sense of the different kinds of actions is also a recognition of the different types of language involved in action. In Act III: Forms of Embodiment, Lahire positions himself against a strand of sociology which sees embodiment as a silent process, separate from language, and against those linguists who forget that language is embodied action. (163) Analysing Wacquant's work on boxing, Lahire stresses the 'polymorphic and multifunctional presence of language' in physical practices: language is embedded in action, not an 'add-on', and is not extricable from the gestures and movements that, together with a variety of forms of language, constitute action. (168-9) The next step is to pose the problem of 'what exactly is embodied?' How, for example, can 'social structures' be embodied, as Bourdieu suggests? Lahire points out that is it particular habits-'schemes of action, ways of doing, thinking, feeling and saying'-that are adapted to each context. (176)

The last Act offers commentaries on 'Workshops and Debates', such as the relationship between sociology and the subjective, and the methodological issues that arise from this interface. He repeats his advocacy of a 'psychological sociology', arguing that if the social sciences are to use cognitively orientated notions of 'disposition' or 'mentality', they have to engage more systematically with consciousness but without sacrificing 'the social' (as some scholars have done) and without resorting to simple oppositions between the objective and the subjective. (195) Unsurprisingly, given his focus on a sociology of individuals, he warns against the tendency to reduce the social to differences between large groups such as classes. (203) He argues that any individual is the product of multiple operations of folding and is characterised by the complexity of social processes, such that the 'interior' is an 'exterior' that is folded. The solution, he claims, is 'to study the "outside" in its finest grain'. (205) The social in an 'unfolded state' is a abstracted generalisation which de-particularises description. In its folded state it is a nuanced combination of contextual and dispositional properties. (vx) This has consequences for the problem of social determination, but it also comes with a methodological imperative to study individuals across multiple settings over time. (209) Along the way he rails against 'empirical laziness' (210) and 'excessive generalisation' (211) and argues for a historicising of sociological analysis. (218) To conclude, Lahire offers several 'lessons' from his discussion of the socially and historical specificity of 
theory-I won't tell you what they are, but they do lead him to make a claim for theoretical modesty and sociological passion.

As a dialogue with Bourdieu, this book will no doubt appeal to devotees of his work, but it is much more than that and as a critical dialogue it would be useful to anyone engaging with the relationship between theory and empirical research, and anyone who would be rejuvenated by a fresh take on the classic questions of social and cultural analysis. Lahire is neither a micro-sociologist who privileges individuals, nor a proponent of the view that macro-social categories like class don't exist. Nor is he claiming to be less 'determinist'; rather, he is emphasising the multidetermined nature of social existence and the 'historical necessity of conceiving the social in a strongly individualising society'. (xviii) Far from rehashing the difficult language of Bourdieu, Lahire seeks to avoid 'enclosing oneself in the endless repetition of pre-established concepts and arguments'. (viii-ix) And he writes better than Bourdieu often did (or is perhaps better translated).

The references to the social sciences and 'scientific thinking' might be disconcerting for cultural studies readers, but Lahire's work speaks to a wider, interdisciplinary audience. Indeed, it speaks directly to that cultural theory which privileges multiplicity, complexity and fluidity, but which rarely develops an analytical approach capable of capturing this dynamism. Given his critical reading of Bourdieu, Lahire is no acolyte. He draws on a range of thinkers-Bergson, Freud, Merleau-Ponty, Goffman, Elias, Bakhtin, Piaget and others-to develop his critique of and extend Bourdieu's insights. Yet it is odd that in a work which stresses the 'actor' that there is no reference to Latour-strangely he says it is unusual to use the notion of actor in French sociology (2) and complains of the demarcation between philosophy and sociology. (5)

Those with a structuralist inclination will complain there is a weak sense of structure, others will complain there is not enough about gender and ethnicity. To do so would downplay the value of this work. But I have one gripe: Lahire doesn't fulfil the promise to develop an understanding of the pedagogic nature of cultural practices. He is aware of limitations of Bourdieu's model of social reproduction, (175) and goes a long way towards recognising the temporal and routine aspects of 'socialisation', but he can't quite shake free of the Bourdieuian frame of transmission he criticises. (178) Lahire begins to enunciate a framework for thinking pedagogy by 
distinguishing between material inheritance and cultural transmission: the latter, he argues, has a longer, gradual timeframe, involves repetition and exercise, has an unconscious element, and is polymorphic. (179-80) Lahire couldn't be expected to solve what has long been a problem, or an absence, in social theory, but the promise is tantalising.

Greg Noble is associate professor at the Institute for Culture and Society, University of Western Sydney. His research focuses on youth, ethnicity and gender; intercultural relations and cosmopolitanism; embodiment and material culture; and multicultural education. His most recent co-authored book is On Being Lebanese in Australia (2010). 